\title{
Manufacturing a chain logically starts with manufacturing the first link, thus is logically started a metrological traceability chain
}

\author{
Paul De Bièvre
}

Published online: 16 September 2009

(C) Springer-Verlag 2009

Many people still struggle with the concept 'metrological traceability' and have trouble when attempting to define it. Yet, it is basically simple: metrological "trace" ability is about our ability to demonstrate the "trace" along which we can show how our measurement result is connected to a commonly agreed reference. Since a measurement is always a comparison, we need a reference against which we can perform such a comparison. That reference can be: (a value of) a measurement unit, a measured value of a measurement standard (calibrator, mostly called a CRM), or a measured value obtained by a commonly agreed reference measurement procedure, along a "track" or "pathway" consisting of a transparent, unbroken chain of links. Hereby, 'calibrator' is meant to be a material, not a person.

As for all 'concepts' we use in our thinking, it is essential for our mutual understanding that we have the same concept in mind for the same thing we are reflecting on. So it is for the concept 'metrological traceability' [1] of a measurement result.

Let us see how said "trace" is manufactured almost automatically when we start measuring, and identify the very operation which manufactures the first link of the "trace".

We do that by means of a simple example: measuring 'mass' (one member of the big family of things we intend to measure, i.e. 'measurands') of, e.g. meat (note: we use single quotation marks to designate a concept). The purpose of measuring is to assign a numerical value

P. De Bièvre $(\square)$

Kasterlee, Belgium

e-mail: paul.de.bievre@skynet.be to that measurand, i.e. to the 'mass' of the meat. We do that by comparing the mass of the meat to a known value of the same measurand (i.e. mass) carried by-or embodied in - a piece of metal with a known mass, called a "weight". Such a known value in a carrier (which can be, among others, a simple material or a complex "matrix" material) is our 'reference'. So, in this example, the operation of measurement consists of assigning a value to a measurand, (the mass of the meat) derived from a comparison to a known value of the same quantity, embodied in a 'reference' (carrier here called a "weight").

To perform that measurement, we build or purchase a measuring system-in the above example, a balancewhich enables us to carry out the comparison to the "reference'. Usually such a reference is called a Certified Reference Material [2], but the (better) generic name is 'measurement standard' [3] or 'calibrator' [4]. The whole process is called a calibration and the 'measurement standard' or 'CRM' acts as a 'calibrator' [4] of our measuring system (the balance). We can then use this "calibrated" measuring system (the balance) to assign a value to the measurand (the mass of meat) which is embodied in the material sample (the meat) under investigation.

Measurement finished. End of story.

Note: it is useful to consider that 'measurement standard' is a more generic concept, i.e. the name of a family of concepts of which 'calibrators' and 'CRMs' are members.

Without thinking about it, we have in fact established the first link of the metrological traceability chain of our measurement result. This first link consists of the bridge built by comparing the value embodied in our sample and previously unknown, to the known value embodied in a measurement standard. The traceability chain of the quantity value embodied in such a calibrator is a matter of 
"higher up" in the traceability chain. The adjective "metrological" is essential to distinguish it clearly from other kinds of - more generic forms of - 'traceability': sample traceability, document traceability, material traceability, etc.

When a calibrator is purchased by the analyst, it is the duty of the seller to deliver the metrological traceability of the certified value embodied in this calibrator in an accompanying certificate, with special emphasis on the metrological traceability of that value to another reference through a next link - the second link-in the metrological traceability chain of our measurement result. This further part of the chain will be considered in a next Editorial.

The analyst should realize that, in fact, (s)he pays for the value (with associated uncertainty), embodied in the calibrator delivered by its producer. This value (with associated uncertainty) constitutes the 'reference' to which the metrological traceability of our measurement result is established and should be mentioned in our reporting of such result.

Since the analyst decides on the measurement standard (CRM) (s)he will use, it is obvious that many kinds of metrological traceability chains are possible and can be established: in general, various measurement standards (CRMs) can be found on the market to serve as 'references' in the same measurement task. They all enable to establish metrological traceability to a reference, which must then be stated clearly. The reason that the analyst must report such a reference is that only measurement results traceable to the same reference, can be compared ("are metrologically comparable") [5] to each other. Such a comparison with measurement results obtained by others for the same measurand in the same or similar material is one of the very reasons why we want to perform measurements.

In all of this we have limited ourselves to the analyst's immediate perspective which is the calibrator, i.e. the measurement standard (s)he purchases. As a rule, (s)he does not make a calibrator him- or herself as (s)he does not have the time or resources to do so. Usually, but not always, a choice of calibrators is available, therefore a choice of (first links of) traceability chains is available, and... possible. It is just a matter for the analyst of choosing and deciding.

The analyst can also decide to go for a 'primary measurement standard' [6] (i.e. a 'primary calibrator'), thus making the metrological traceability chain very short: the analyst's calibrator can contain a known submultiple of a mole of the substance concerned, "embodied" in either a near-pure material or in a matrix material because prepared rather than measured, in order to meet the definition of 'primary measurement standard' [6].

Example: we mix a known amount of substance, say glucose, with a known mass of water, calculate the glucose content of the solution and express it as a content, an amount (of glucose) per mass (of solution). An uncertainty is associated with this content because we cannot determine perfectly either the amount of glucose dissolved or the mass of water. We have embodied a known value (with associated uncertainty) of the glucose content in a material: a glucose solution. We know the value of the content from the preparation parameters, which means obtained not from a measurement of the content of glucose in water, but from the preparation of the solution. It is therefore a primary calibrator (or 'primary measurement standard') for the chemical measurement of glucose content in water since it meets the definition of 'primary measurement standard' [6]. Note that the metrological traceability chain of the quantity value for 'amount per mass' embodied in a material in this example must be traceable to other calibrators for the quantity concerned (amount of substance per mass). These usually have much smaller, in fact negligible, measurement uncertainties, another very reason for using this approach.

Starting a metrological traceability chain is relating a measurement result to a stated reference along a "trace" or "pathway" involving one transparent operation. That reference can be a measured value embodied in a purchased 'working measurement standard', i.e. a 'working calibrator' (which implies one or more further links in the traceability chain, thus making it longer-see next Editorial), or it can be a 'primary measurement standard', i.e. a 'primary calibrator', either purchased or made up by the analyst him/herself. In both cases, the metrological traceability chain consists of only one link to a 'reference'. It is preferable that the working calibrator was implicitly or explicitly agreed on beforehand as 'reference', otherwise it cannot fulfill a useful role as common reference. In the case of a 'primary measurement standard', that common agreement is already there since we have agreed on a common definition (i.e. in the VIM).

Similarly, a measured value obtained by a 'reference measurement procedure' [7]—which has to be commonly agreed on beforehand as delivering a 'reference'-can serve as 'reference' to establish metrological traceability of measurement results obtained by other measurement procedures. Such a measurement procedure can even be an 'operationally defined measurement procedure' to measure an 'operationally defined measurand'. Also in this case, the metrological traceability chain consists essentially of one link only.

Simple and clarifying.

As basic metrological concepts always should be...

The possibility of using a 'reference measurement procedure' as reference has been created by VIM3 and enables to establish comparability of all measurement results obtained for the same measurand in the same matrix 
material by a 'reference measurement procedure' when a measurement unit or an appropriate measurement standard (calibrator or CRM) is not (yet) available. It may be the subject of another Editorial.

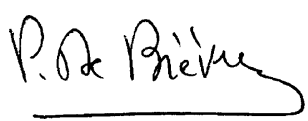

Paul De Bièvre

Editor-in-Chief

\section{References}

1. BIPM, IEC, IFCC, ILAC, IUPAC, IUPAP, ISO, OIML (2008) The international vocabulary of metrology - basic and general concepts and associated terms (VIM), 3rd edn. JCGM 200. http://www. bipm.org/en/publications/guides/vim.html, entry 2.41

2. Ibidem, entry 5.14

3. Ibidem, entry 5.1

4. Ibidem, entry 5.12

5. Ibidem, entry 2.46

6. Ibidem, entry 5.4

7. Ibidem, entry 2.7 